\title{
Geodetic and direct mass-balance measurements: comparison and joint analysis
}

\author{
J. Graham COGLEY \\ Department of Geography, Trent University, Peterborough, Ontario, K9J 7B8, Canada \\ E-mail: gcogley@trentu.ca
}

\begin{abstract}
This paper describes a new compilation of both direct and geodetic mass-balance measurements, develops a procedure to reduce diverse balance measurements over different time-spans to common time-spans, and presents updated estimates of global average balance of small glaciers based on the enlarged compilation. Although geodetic measurements are fewer than direct measurements, they cover four times as many balance years. Direct and geodetic measurements are unbiased with respect to one another, but differences are often substantial. The statistical procedure can be understood by imagining that an $n$-year balance measurement is an average of a series of 1 year measurements. The series is hypothetical but we can calculate the uncertainty of each of its elements if, in addition to its measured average, we can also estimate its standard deviation. For this claim to be valid, the annual series must be stationary and normally distributed with independent (roughly, uncorrelated) elements, for which there is reasonable evidence. The need to know the standard deviation means that annual direct measurements from a nearby glacier, or equally reliable information about variability, are indispensable. Given this information, the new methodology results in moderately more negative balances. This is probably because tidewater glaciers are better represented in the geodetic data. In any case, the most recent published estimate of global average balance, $0.8-1.0 \mathrm{~mm} \mathrm{a}^{-1}$ of sea-level equivalent for 2001-04, is now increased substantially to $1.1-1.4 \mathrm{~mm} \mathrm{a}^{-1}$ for 2001-05.
\end{abstract}

\section{INTRODUCTION}

Global averages of glacier mass balance (Kaser and others, 2006) are based mostly on direct measurements, which support reasonably convincing estimates at annual (Ohmura, 2004; Dyurgerov and Meier, 2005) or pentadal (Cogley, 2005) resolution. These data leave much to be desired in terms of coverage. One way to improve coverage would be to draw on geodetic as well as direct measurements. However, before we can treat direct and geodetic balance measurements within the same analytical framework, we must address the mismatch in temporal resolution. Direct measurements are nearly all annual, whereas geodetic measurements are nearly all multi-annual. The aims of this paper are therefore to: (1) describe a new compilation of geodetic mass-balance measurements; (2) compare all available direct-geodetic measurement pairs so as to identify possible biases; (3) develop a common analytical framework for diverse balance measurements; and (4) present updated estimates of global balance based on the new compilation.

For compactness, annual whole-glacier mass balance is denoted here as $b$, without distinguishing the method of measurement, and as $d$ or $g$ when we need to make clear that the measurement is direct or geodetic, respectively. Estimates for $n$-year spans, with $n \geq 1$, are denoted by $B, D$ or $G$, and variances by $\sigma_{i}^{2}$, with $i$ standing for $b, d$ or $g$.

\section{AVAILABLE DATA}

Geodetic balance measurements were assembled from just over 100 published sources. Most are single; only $40 \%$ of the glaciers have more than one measurement. The distribution of measurement spans is very wide, with many covering several decades. However, the number of glaciers with geodetic measurements is not very different from the number with direct measurements (Table 1). There are fewer than one-fifth as many geodetic as direct measurements, although they cover four times as many balance years. They also cover more calving glaciers, in proportions likely to be closer to the (unknown) ideal, than do direct measurements. Two-fifths of glaciers with more than one geodetic measurement probably exhibit more rapid loss recently than earlier, a somewhat smaller fraction than for glaciers with direct measurements.

The main message of Table 1, reinforced by Figure 1, is that we could realize a dramatic increase in data density, measured in balance years, if we could remedy the absence of information about temporal variability in multi-annual measurements.

Figure 2 summarizes the evolution of mass balance since 1860. Figure 2a confirms that mass balance has recently become more negative, although this might result from wider geodetic sampling. Figure $2 \mathrm{~b}$ is subject to the same possible sampling problems as Figure $2 \mathrm{a}$ but, at face value, it is encouraging because, despite the smoothing of $G$, the two methods agree in showing a relative maximum near 1970 and an accelerating decrease since then. However, $G$ is distinctly more negative than $D$, which should lead us to expect reduced estimates when the two are combined and corrected for spatial bias. A recent estimate of the evolution of mass balance since the 19th century (Oerlemans and others 2007), based on measurements of terminus fluctuations, lies in between the arithmetic-average series of $D$ and $G$, which strengthens this expectation.

\section{TREATMENT OF DATA}

The essential attributes of the direct-balance dataset have not changed since it was first described by Cogley and Adams (1998). I concentrate here on the newly assembled 
Table 1. Comparison of direct and geodetic mass-balance datasets

\begin{tabular}{lcc}
\hline Attribute & Direct & Geodetic \\
\hline Number of glaciers* & 344 & 327 \\
Number of measurements & 4039 & 754 \\
Number of balance years* & 4146 & 16383 \\
Marine-calving glaciers (\%) & 3 & 16 \\
Lake-calving glaciers (\%) & 4 & 16 \\
Most recent balance minus next most & $54 / 40 / 6$ & $40 / 46 / 14$ \\
recent ${ }^{\dagger}$ (\%) & & \\
Information about interannual variability & $\propto N_{y}{ }^{2}$ & nil \\
\hline
\end{tabular}

*59 glaciers, and a total of 1052 balance years, appear in both datasets. Geodetic column includes all $n$-year measurements $(n \geq 1)$.

The three numbers in each column are percentages of differences which are respectively negative, within \pm 1 standard error of 0 , and positive; for direct data, successive pentadal average balances are subtracted.

geodetic data. In practice, there being seldom any in situ information about density, geodetic measurements approximate the average of specific mass change over time-span $\Delta t$ by:

$$
G=\bar{\rho} \overline{\Delta h} / \Delta t
$$

Glacier-wide average density $\bar{\rho}$ is usually assumed to be $900 \mathrm{~kg} \mathrm{~m}^{-3}$. Lesser values are sometimes adopted, particularly when decreases of thickness are believed to involve losses of firn rather than of ice. I accept the authors' value for $\bar{\rho}$ when one is given and assume that it is $900 \mathrm{~kg} \mathrm{~m}^{-3}$ when no value is given. A rare study in which uncertainties in $\bar{\rho}$ are considered is that of Sapiano and others (1998). I adopt their suggested error of about $6 \%$ in mass balance due to the uncertainty in density.

Various methods, differing greatly in intrinsic accuracy and in completeness of sampling, have been applied to the estimation of areal-average thickness change $\overline{\Delta h}$. The traditional method was to compute changes of elevation from contours on printed maps. Recently, it has become usual to take differences of digital elevation models or to extrapolate from surveyed traverses. When a standard error is given for $\overline{\Delta h}$ and is plausible, it is adopted without change; otherwise, a standard error of $\pm 3.0 \mathrm{~m}$ is assumed. This is comparable with the uncertainties published by workers who have made careful analyses of their errors, and also allows for the lack of published information about seasonal corrections, the difficulty of mapping elevation over featureless snowfields and, for some methods, the uncertainty resulting from incomplete sampling. As is appropriate to the nature of the measurements, the error is not proportional to the amount of time between start and end surveys. The practice of representing glacier area by the average of starting and ending glacier areas, which is common but not invariable, is followed uniformly.

The time-span can be a source of significant uncertainty, especially when dates of early photography are in question or when imagery from more than one date is used to represent a single epoch. I record the uncertainty as a range, in days, and assume that the standard error of each date is half of the range. Thus, for example, dates given only to the nearest year have an uncertainty of half a year. Seasonal corrections are necessary when survey dates are not separated by an integer number of years. They restore the

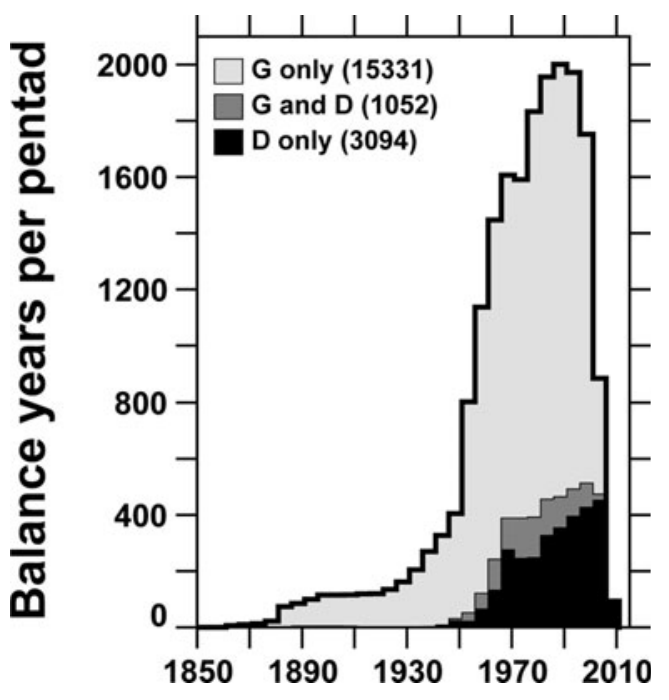

Fig. 1. Distribution of geodetic $(G)$ and direct $(D)$ measurements over time. Each geodetic measurement contributes up to five balance years to each of the pentads which it spans.

average surface elevation to a value appropriate to the beginning or end of the balance year, or to the date of the other survey. When given, they are adopted without change, except that they are assigned an uncertainty of $\pm 20 \%$ if necessary. It is not practical to supply seasonal corrections when they are not given by authors. Indeed, it is often not possible to tell whether they are needed. It is commonly assumed that seasonal corrections are not necessary when the start and end dates are far apart. In fact, they become insignificant when measured thickness changes are large by comparison with the amplitude of the seasonal cycle of thickness, which is not the same thing.

The uncertainty in $G$ is calculated, based on Equation (1), by conventional propagation of the standard errors of $\bar{\rho}, \overline{\Delta h}$ and $\Delta t$.

The standard error of annual $d$ is estimated to be $200 \mathrm{~kg} \mathrm{~m}^{-2} \mathrm{a}^{-1}$, following Cogley and Adams (1998). This error grows at $10 \% \mathrm{a}^{-1}$ for those few measurements that are multi-annual.

\section{DIRECT-GEODETIC COMPARISONS}

Figure 3 shows that there is no evidence, at this scale of analysis, of systematic differences between $D$ and $G$. The average difference, $-74 \mathrm{~kg} \mathrm{~m}^{-2} \mathrm{a}^{-1}$ or -0.09 standard errors, is negligible, but the spread is substantial. The root mean square of $G-D$ is $378 \mathrm{~kg} \mathrm{~m}^{-2} \mathrm{a}^{-1}$, but only $3 \%$ of the differences, all with $D$ greater than $G$, are statistically significant. The best-fitting linear relationship between $D$ and $G$ has a slope (not shown) of $\mathrm{d} D / \mathrm{d} G=0.85 \pm 0.10$, indicating that very negative $G$ is associated with somewhat less negative $D$. (Unless stated otherwise, uncertainties are quoted as twice the standard error.) A physical explanation of this result remains to be identified, although it might be connected with the better representation of tidewater glaciers in the geodetic dataset. Much effort has gone into seeking explanations for observations of $G-D$. The new compilation offers an opportunity to assess reasons for the observed differences and to decide whether they have a physical rather than an instrumental basis; however, a more detailed approach than is possible here will be needed. 

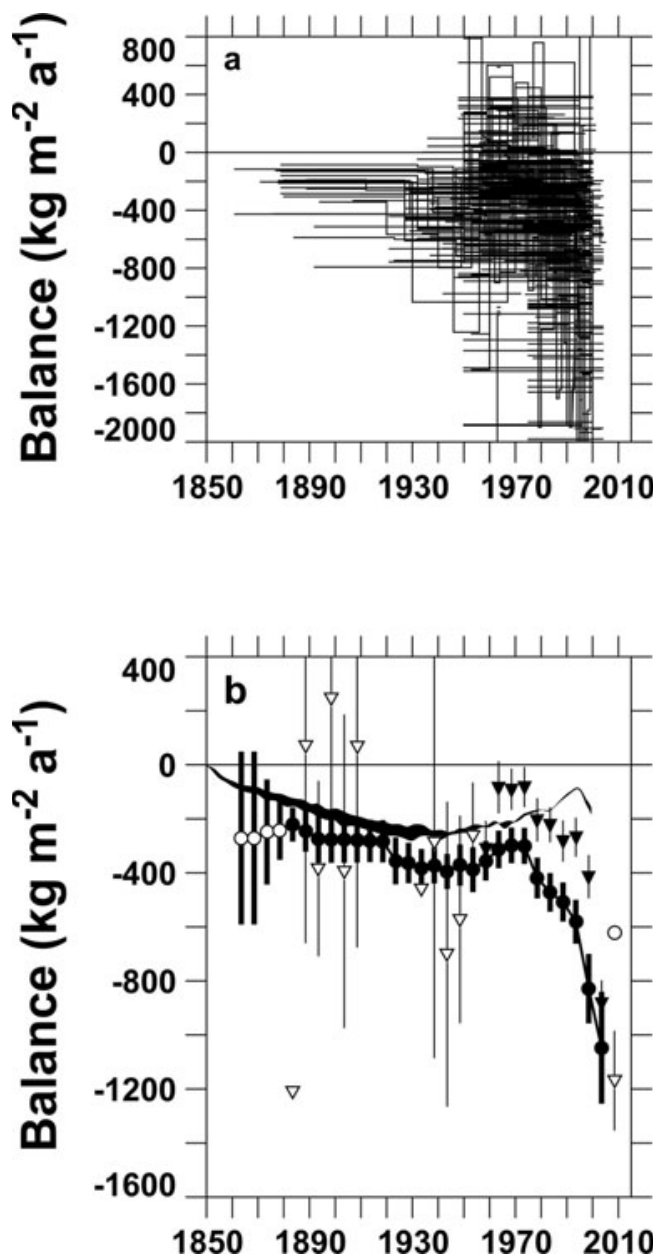

Fig. 2. Evolution of geodetically measured mass balance. (Note different balance-axis scales.) (a) The measurements for each glacier are plotted as a time series; 30 of 678 measurements lie beyond the range of the balance axis. (b) Pentadal arithmetic averages over all measured glaciers of $G$ (circles, open for pentads with fewer than 10 measurements) and $D$ (triangles, open for pentads with fewer than 100 annual measurements). Multi-annual measurements are counted in each of the pentads which they span. Vertical bars represent \pm 2 standard errors of each pentadal sample about its arithmetic average. The irregular dark grey envelope is a rendering of the estimates of Oerlemans and others (2007, fig. 7).

The open symbols in Figure 3 represent measurements spanning only 1 or 2 years. In the dataset as a whole, there are 40 such measurements of $G$, with an average standard error of $1372 \mathrm{~kg} \mathrm{~m}^{-2} \mathrm{a}^{-1}$. This means that, in practice, they are not useful. Typical errors in $\overline{\Delta h}$ and $\bar{\rho}$ limit the temporal resolving power of geodetic methods to no better than $\sim 3$ years

\section{VARIANCE ESTIMATION FOR MULTI-ANNUAL BALANCE MEASUREMENTS}

An $n$-year balance measurement can be seen as an average of a series of hypothetical annual measurements. It can also be seen as an estimate of each actual 1 year balance. The error due to natural variability, that is, the uncertainty in each annual balance $g$ (estimated to be equal to $G$ ), is the standard deviation of the population of which the hypothetical series is a sample. For this claim to be valid, the annual series must be stationary and normally distributed

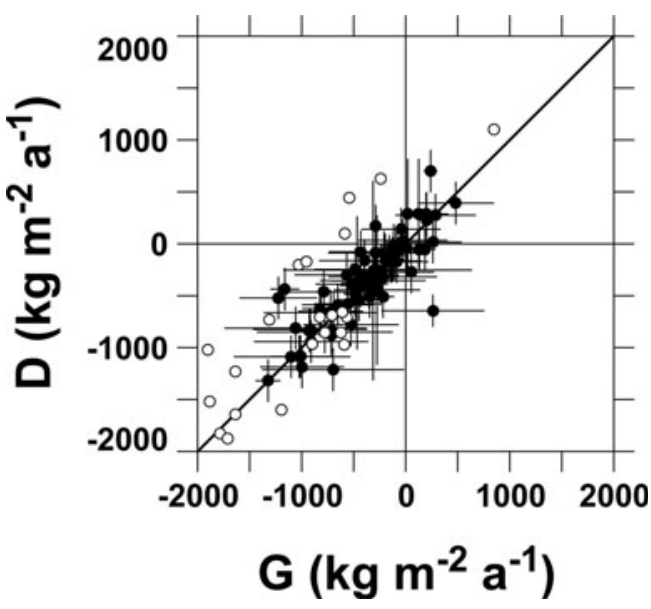

Fig. 3. Relationship between 105 coincident geodetic and direct mass-balance measurements, $G$ and $D$ respectively, from 29 glaciers. Five pairs, all with large uncertainties in $G$, lie beyond the range of the axes. Error bars represent \pm 1 standard error. Open symbols: measurements shorter than 3 years.

with independent (roughly speaking, uncorrelated) elements. There is reasonable evidence that these conditions hold for mass-balance time series.

To estimate the standard deviation of any given geodetic balance, I propose to use the relation:

$$
\sigma_{g}=\sigma_{0}+\frac{\mathrm{d} \sigma}{\mathrm{d} T_{\mathrm{w}}} T_{\mathrm{w}}
$$

where $\sigma_{0}$ and $\mathrm{d} \sigma / \mathrm{d} T_{\mathrm{w}}$ are parameters to be estimated. Equation (2) specifies a linear dependence of interannual variability on the temperature $T_{\mathrm{w}}$ at the glacier's equilibriumline altitude (ELA) during the warmest month of the balance year. This choice is the outcome of an extensive exploration of the direct-balance data for possible influences on interannual variability, $\sigma_{d}$. For example $\sigma_{d}$ is uncorrelated with the mass balance itself and with record length, although the spread of observed values is greater the shorter the record. There is a weak inverse correlation with glacier size, and the horizontal separation between measured glaciers is not a good predictor of $\sigma_{d}$ (Fig. 4a).

Figure $4 \mathrm{a}$ is based on the standard error $\mathrm{se}_{\sigma}=\left|\sigma_{d}(A)-\sigma_{d}(B)\right| / 2$ of pairs of standard deviations calculated for all glacier pairs $(A, B)$. The $\mathrm{se}_{\sigma}$ are sorted by $s$ (the distance between $A$ and $B$ ) and grouped into successive sets, along the $s$ axis, of 40 measurement pairs each. The grey envelope, representing \pm twice the standard error of each of these sets, shows that $\mathrm{se}_{\sigma}$ increases very slowly with separation. Thus nearby glaciers with reliably known $\sigma_{d}$ offer no particular advantage over distant ones for estimating $\sigma_{g}$.

In Figure $4 b$, however, the variability depends recognizably on the continentality, glaciers with colder ELAs being more 'continental'. The best-fit line (Equation (2)) explains $29 \%$ of the sample variance. The data are scattered, but the relationship is well defined enough to require that it be allowed for. Accordingly, I adopt Equatin (2) as the source of information for estimating $\sigma_{g}$.

This is consistent with Braithwaite and Zhang (1999), who showed that the interannual variability of mass balance scales with the balance amplitude (one-half of the difference between winter balance and summer balance). However, 

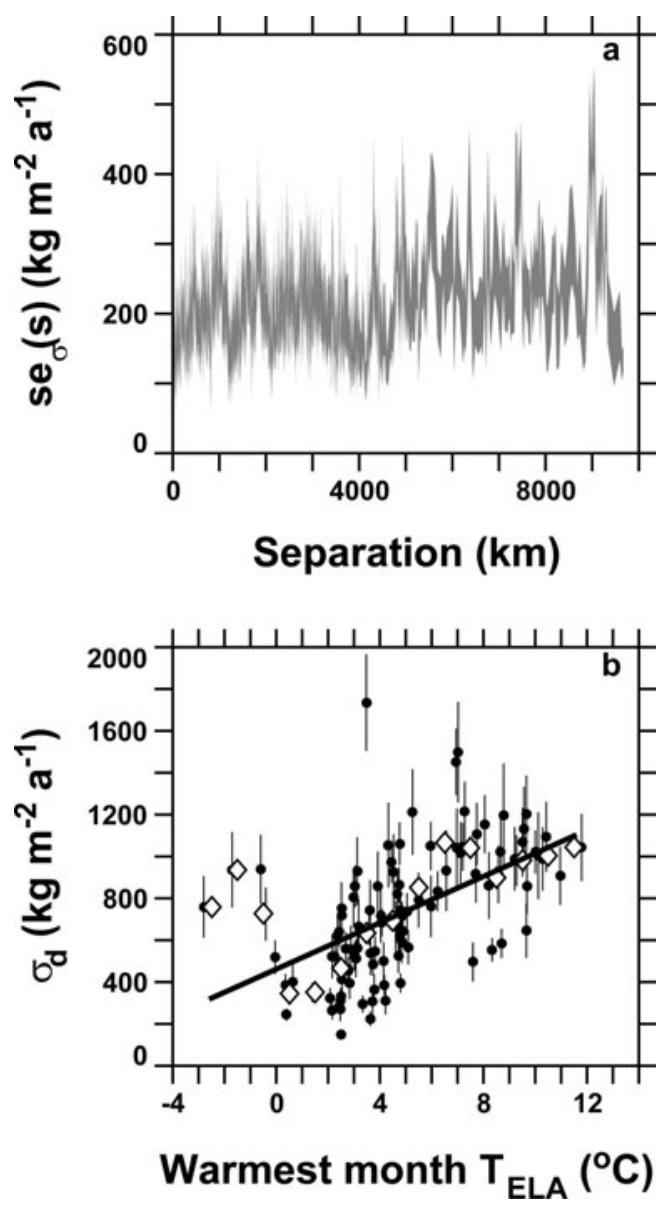

Fig. 4. Possible determinants of interannual variability of mass balance. (a) Predictability of standard deviation from pairs of directbalance standard deviations separated by distance $s(\mathrm{~km})$. See text for details of $\mathrm{se}_{\sigma}(\mathrm{s})$. (b) $\sigma_{d}$ for series of $D$ longer than 12 years (selected to avoid unreliable short-record $\sigma_{d}$ ) as a function of $T_{w}$, the free-air temperature at the ELA during the warmest month (see text for details). Best-fit line, $\sigma_{d}=465+55 T_{\mathrm{w}}$, derived by regression against single-glacier observations. Diamonds: class averages (class width $1^{\circ} \mathrm{C}$ ).

seasonal balances are even less widely measured than annual balances, and here a universally available measure of variability is needed. As noted by Braithwaite (2008; see also Ohmura and others, 1992), for any given annual balance a greater amplitude implies both snowier winters and warmer summers.

The quantity $T_{\mathrm{w}}$ is interpolated to each glacier's ELA from the US National Centers for Environmental Prediction-US National Center for Atmospheric Research (NCEP-NCAR) reanalysis (Kalnay and others, 1996) and an unpublished dataset of average ELAs at $1^{\circ} \times 1^{\circ}$ resolution. The reanalysis temperatures are climatological averages for 1968-96. The ELA data come principally from an enlarged version of the World Glacier Inventory (WGI) containing 125000 glaciers (Cogley, 2008). The ELA is estimated by averaging the midaltitudes (averages of minimum and maximum elevations; Cogley and Mclntyre, 2003) of single glaciers. Where the WGI is incomplete, ELAs are estimated from surrogates. For example, much of the information for western North America consists of glaciation levels (e.g. Østrem, 1972), reduced by $200 \mathrm{~m}$ following Cogley and McIntyre (2003).

Following Cogley (2004, section 4.3), the difference due to unsampled natural variability between an $n$-year $G$ and any one of the annual measurements $g$ of which we imagine it to be composed is expected to be zero, with an uncertainty given by

$$
\operatorname{se}_{\mathrm{NV}}=\sqrt{\sigma_{g}^{2}(1+1 / n)}
$$

This, however, is not a complete description of the uncertainty in $g$. The measured $G$ is itself uncertain, and we must allot some of the measurement error to each of the $n$ years in the span of the measurement. I assume that the measurement error se $G$ is composed of equal annual contributions, so that the uncertainty in $g$ due to measurement error is

$$
\operatorname{se}_{\mathrm{ME}}=\operatorname{se} G / n \text {. }
$$

Further, I assume that $\mathrm{se}_{\mathrm{NV}}$ and $\mathrm{se}_{\mathrm{ME}}$ are independent. Combining Equations (3) and (4), this leads to

$$
\operatorname{se}^{2} g=\sigma_{g}^{2}(1+1 / n)+\operatorname{se}^{2} G / n^{2}
$$

as our best estimate of the total uncertainty in each annual $g$.

\section{JOINT ANALYSIS WITH DIRECT AND GEODETIC MEASUREMENTS}

We can now combine 'measurements' of annual $g$ with measurements of annual $d$. Here I calculate global average $B$ in the same way as in earlier work (Cogley, 2005; Kaser and others, 2006). The annual estimates are used to interpolate pentadal averages at each glacierized cell of a $1^{\circ} \times 1^{\circ}$ grid, these averages then being weighted by the glacierized area of the cell. When both $d$ and $g$ are available for any glacier, $d$ is chosen and not $g$. When a set of up to five measurements is a mixture of $d s$ and gs, its uncertainty is calculated as the standard deviation of the sample divided by the square root of $m$, the number of annual measurements. But when only gs are available the uncertainty is $\Sigma\left(\operatorname{se~}_{i}\right) / \mathrm{m}$.

Figure 5 shows the new pentadal averages $B$ in the context of recent estimates that used only direct measurements $D$. In all but the most recent pentads, $B$ is more negative than the uncorrected arithmetic average of direct measurements, and in all but the 1956-60 pentads it is more negative than $D$.

First, however, note that $D$ itself is now more negative than in Kaser and others (2006). Their C05i was $-481 \pm 50 \mathrm{~kg} \mathrm{~m}^{-2} \mathrm{a}^{-1}$ for 2001-04, to be compared with the current $D=-720 \pm 58 \mathrm{~kg} \mathrm{~m}^{-2} \mathrm{a}^{-1}$. This is mainly because of newly assimilated measurements of $d$ for 2005, which was a year of serious mass loss on a global average. As Figure 5 suggests, 2006 was probably even more negative, so incoming data provide no sign that the trend to faster mass loss is abating. The trend since 1971-75 is virtually unchanged $\left(-10.5 \mathrm{~kg} \mathrm{~m}^{-2} \mathrm{a}^{-2}\right)$ in the transition from the $D$ only dataset to the $D$-plus- $G$ dataset.

In 2001-05 $B$, at $-740 \pm 62 \mathrm{~kg} \mathrm{~m}^{-2} \mathrm{a}^{-2}$, is indistinguishable from $D$. This could be because, as yet, there are fewer recent geodetic measurements. More generally, it is not an accurate description of the past 50 years as a whole, and it is probably no longer correct to say that $B$ was near to zero in the 1960 s and early 1970 s.

The small-glacier contribution to sea-level rise is now estimated at $1.12 \pm 0.14 \mathrm{~mm} \mathrm{a}^{-1}$ SLE (sea-level equivalent) for 2001-05, as against the $0.77 \pm 0.15 \mathrm{~mm} \mathrm{a}^{-1}$ SLE of Kaser and others (2006). These numbers exclude glaciers in Greenland and Antarctica. Kaser and others estimated 


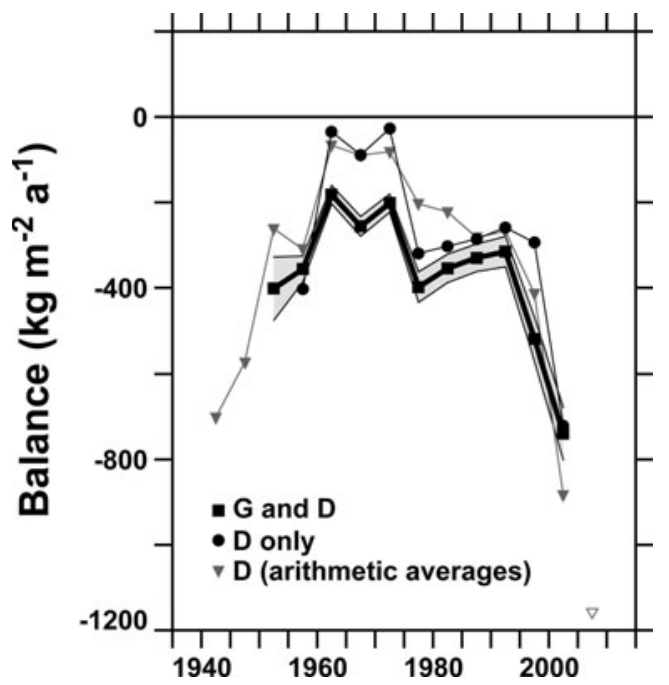

Fig. 5. Pentadal calculations of global average mass balance, updating parts of figure 2 of Kaser and others (2006). Triangles: arithmetic averages of direct balance measurements (C05a of Kaser and others (2006), extended by completion of 2001-05 pentad. Circles: similarly extended averages with spatial interpolation from direct balance measurements to correct for spatial bias (equivalent to $\mathrm{C05i}$ of Kaser and others). Squares: averages with spatial interpolation from the combined direct and geodetic balance dataset introduced in this study, with grey confidence region representing \pm 2 standard errors about the mean.

$0.98 \pm 0.19 \mathrm{~mm} \mathrm{a}^{-1}$ SLE including those glaciers, and taking their approach to upscaling, the equivalent figure for the present study is $1.41 \pm 0.20 \mathrm{~mm} \mathrm{a}^{-1}$ SLE.

\section{CONCLUDING REMARKS}

It seems probable that the global balances presented here are more negative than earlier estimates because of better representation of tidewater glaciers. This suggestion should be explored more thoroughly, as should the question of whether the enlarged dataset does in fact represent adequately the global-average ablation by calving. Note also that no progress has been reported here on the other biases discussed by Kaser and others (2006).

The confidence region shown for $B$ in Figure 5, derived from standard errors of polynomial coefficients estimated during spatial interpolation, is narrower than that of the consensus estimates presented by Kaser and others (2006). If one of the purposes of spatial interpolation is to smooth away errors in the observations, then Figure 5 contains meaningful short-term detail that deserves investigation. The relative minimum in 1975-80 is an example. If, however, the errors of the polynomial coefficients are physically uninformative, an alternative method of estimating uncertainty must be found. Routine cross-validation calculations, in which an estimate of the balance is interpolated at each measured glacier but without the information from that glacier itself, typically yield root-mean-square differences (measured minus fitted) an order of magnitude greater than the errors in Figure 5. This would be much more cautious, but might require us to doubt whether global average mass balance is growing more negative.

Another note of caution should be sounded in conclusion. The error model for $G$ is quite detailed, whereas the error model for $D$, unaltered in the present study, must now be seen as underdeveloped. As it stands, the error analysis favours measurements of $d$, because larger errors are usually assigned to $g$ and the direct data tend to outweigh the geodetic data during averaging. However, the relative error weighting of the two kinds of data could be tuned plausibly within quite broad limits. The crude error model for $d$ needs to be revisited, and the error model for $G$ needs to be placed on a more secure observational footing, which will require better modelling of the interannual variability of mass balance. A considerable amount of work is necessary before the new dataset can be regarded as a source of homogeneous information, but it is likely that our estimates of the global average mass balance of small glaciers are now better.

\section{ACKNOWLEDGEMENTS}

I thank all those colleagues who have published measurements of mass balance, whether by geodetic or direct methods, and the World Glacier Monitoring Service, Zürich.

\section{REFERENCES}

Braithwaite, R. 2008. Temperature and precipitation climate at the equilibrium-line altitude of glaciers expressed by the degree-day factor for melting snow. J. Glaciol., 54(186), 437-444.

Braithwaite, R.J. and Y. Zhang. 1999. Relationships between interannual variability of glacier mass balance and climate. J. Glaciol., 45(151), 456-462.

Cogley, J.G. 2004. Greenland accumulation: an error model. J. Geophys. Res., 109(D18), D18101. (10.1029/2003JD004449.)

Cogley, J.G. 2005. Mass and energy balances of glaciers and ice sheets. In Anderson, M.G., ed. Encyclopaedia of hydrological sciences. Part 14. Snow and glacier hydrology. New York, Wiley, 2555-2573.

Cogley, J.G. and W.P. Adams. 1998. Mass balance of glaciers other than the ice sheets. J. Glaciol., 44(147), 315-325.

Cogley, J.G. and M.S. Mclntyre. 2003. Hess altitudes and other morphological estimators of glacier equilibrium lines. Arct. Antarct. Alp. Res., 35(4), 482-488.

Dyurgerov, M.B. and M.F. Meier. 2005. Glaciers and the changing Earth system: a 2004 snapshot. Boulder, CO. Institute of Arctic and Alpine Research. (INSTAAR Occasional Paper, 58.)

Kalnay, E. and 21 others. 1996. The NCEP/NCAR 40-year reanalysis project. Bull. Am. Meteorol. Soc., 77(3), 437-471.

Kaser, G., J.G. Cogley, M.B. Dyurgerov, M.F. Meier and A. Ohmura. 2006. Mass balance of glaciers and ice caps: consensus estimates for 1961-2004. Geophys. Res. Lett., 33(19), L19501. (10.1029/2006GL027511.)

Oerlemans, J., M. Dyurgerov and R.S.W. van de Wal. 2007. Reconstructing the glacier contribution to sea-level rise back to 1850. Cryosphere, 1(1), 59-65.

Ohmura, A. 2004. Cryosphere during the twentieth century. In Sparling, J.Y. and C.J. Hawkesworth, eds. The state of the planet: frontiers and challenges in geophysics. Washington DC, American Geophysical Union, 239-257.

Ohmura, A., P. Kasser and M. Funk. 1992. Climate at the equilibrium line of glaciers. J. Glaciol., 38(130), 397-411.

Østrem, G. 1972. Height of the glaciation level in northern British Columbia and southeastern Alaska. Geogr. Ann., 54A(2), 76-84.

Sapiano, J.J., W.D. Harrison and K.A. Echelmeyer. 1998. Elevation, volume and terminus changes of nine glaciers in North America. J. Glaciol., 44(146), 119-135. 